THE DENSITY OF THE TIME TO RUIN

IN THE CLASSICAL POISSON RISK MODEL

BY

David C.M. Dickson And Gordon E. Willmot

\begin{abstract}
We derive an expression for the density of the time to ruin in the classical risk model by inverting its Laplace transform. We then apply the result when the individual claim amount distribution is a mixed Erlang distribution, and show how finite time ruin probabilities can be calculated in this case.
\end{abstract}

\title{
KEYWORDS
}

Time to ruin, Laplace transform, Lagrange's implicit function theorem, Compound geometric distribution, Mixed Erlang distribution, Finite time ruin, Transient M/G/1 waiting time.

\section{INTRODUCTION AND NOTATION}

In the classical risk model, the insurer's surplus process $\{U(t)\}_{t \geq 0}$ is given by

$$
U(t)=u+c t-S(t)
$$

where $u$ is the insurer's initial surplus, $c$ is the rate of premium income per unit time, and $S(t)$ is the aggregate claims outgo up to time $t$. Further, $S(t)=$ $\sum_{i=1}^{N(t)} X_{i}$ (with $S(t)=0$ when $N(t)=0$ ) where $\{N(t)\}_{t \geq 0}$ is a Poisson process with parameter $\lambda$, and $\left\{X_{i}\right\}_{i=1}^{\infty}$ is a sequence of independent and identically distributed random variables, independent of $\{N(t)\}_{t \geq 0}$, with $X_{i}$ representing the amount of the $i$ th individual claim. Let $P(x)=\operatorname{Pr}\left(X_{1} \leq x\right)=1-\bar{P}(x)$ be the distribution function of individual claims. We assume throughout that $P$ has a density function $p$, and that $P(0)=0$ so that all claim amounts are positive. We use the notation $\tilde{p}$ to denote the Laplace transform of $X_{1}$, so that

$$
\tilde{p}(s)=\int_{0}^{\infty} e^{-s x} p(x) d x .
$$

Let $m_{1}$ denote the mean individual claim amount, and assume that $c=(1+\theta) \lambda m_{1}$ where $\theta>0$ is the premium loading factor. 
At this stage it is convenient to introduce the equilibrium distribution of $P$ which we denote by $P_{1}$, with associated density $p_{1}(x)=\bar{P}(x) / m_{1}$, and Laplace transform $\tilde{p}_{1}$.

Define $T_{u}$ to be the time to ruin (from initial surplus $u$ ) so that $T_{u}=\inf \{t$ : $U(t)<0\}$, with $T_{u}=\infty$ if $U(t) \geq 0$ for all $t>0$. We denote the ultimate ruin probability from initial surplus $u$ by $\psi(u)$, so that $\psi(u)=\operatorname{Pr}\left(T_{u}<\infty\right)$, and denote the finite time ruin probability by $\psi(u, t)$, so that $\psi(u, t)=\operatorname{Pr}\left(T_{u} \leq t\right)$. Clearly $T_{u}$ is a defective random variable with (defective) density

$$
w(u, t)=\frac{\partial}{\partial t} \psi(u, t) .
$$

We can alternatively consider the proper random variable $T_{u, c}$ defined as $T_{u, c}=$ $T_{u} \mid T_{u}<\infty$ with proper density function $w_{c}(u, t)=w(u, t) / \psi(u)$.

In recent years, there has been considerable interest in the moments and distribution of the time to ruin in the classical risk model. For example, Lin and Willmot (2000) present a recursion scheme from which moments of $T_{u}$ can be found, while Drekic et al (2004) discuss evaluation of these moments. Dickson and Waters present a numerical approach to finding the density of $T_{u, c}$, while Garcia (2002), Drekic and Willmot (2003) and Dickson et al (2003) all consider inversion of the Laplace transform of $T_{u}$. In this paper we pursue the latter approach in order to derive an expression for the density of $T_{u}$.

This paper is organised as follows. In the next section we set out some properties of the Laplace transform of $T_{u}$. Then in Section 3 we consider the situation when $u=0$ and find an expression for the density of $T_{0, c}$ by first deriving an important transform identity. In Section 4 we consider the more general situation when $u>0$, and we conclude with an illustration of our main result in Section 5 in the case when the individual claim amount distribution is a mixture of Erlangs. In this special case, an expression for $\psi(u, t)$ then follows, and this may be interpreted in terms of the transient waiting time distribution in the M/G/1 queue. See, for example, Asmussen (2000, Section V.4).

\section{THE LAPLACE TRANSFORM OF $T_{u}$}

Central to the subsequent analysis is Lundberg's fundamental equation, given by

$$
\lambda+\delta-c s=\lambda \tilde{p}(s),
$$

where $\delta$ is a non-negative parameter. Gerber and Shiu (1998) show that this equation has a unique positive solution which, for brevity, we denote by $\rho$, rather than the more cumbersome $\rho(\delta)$.

We now consider the function

$$
\phi_{\rho}(u)=E\left[e^{-\delta T_{u}} I\left(T_{u}<\infty\right)\right]
$$

where $I\left(T_{u}<\infty\right)=1$ if $T_{u}<\infty$ and is zero otherwise. It is well known (see Gerber and Shiu (1998)) that the function $\phi_{\rho}$ depends on $\delta$ solely through $\rho=\rho(\delta)$, and it is convenient to adopt the present notation. This function is actually a 
special case of a more general function introduced by Gerber and Shiu (1998), and we will treat it as the Laplace transform of the time to ruin.

Lin and Willmot (1999) show that $\phi_{\rho}(u)$ has a compound geometric representation, namely

$$
\phi_{\rho}(u)=\sum_{n=1}^{\infty}\left(1-\phi_{\rho}(0)\right)\left(\phi_{\rho}(0)\right)^{n} \bar{G}_{\rho}^{n *}(u)
$$

where

$$
\begin{aligned}
& \phi_{\rho}(0)=\psi(0) \tilde{p}_{1}(\rho)=\frac{\lambda m_{1}}{c} \tilde{p}_{1}(\rho), \\
& G_{\rho}(x)=1-\frac{\int_{x}^{\infty} e^{-\rho(y-x)} \bar{P}(y) d y}{m_{1} \tilde{p}_{1}(\rho)},
\end{aligned}
$$

and $G_{\rho}^{n *}=1-\bar{G}_{\rho}^{n *}$ is the $n$-fold convolution of the distribution function $G_{\rho}$ with itself. Further,

$$
\frac{d}{d x} G_{\rho}(x)=\frac{1}{m_{1} \tilde{p}_{1}(\rho)} \int_{x}^{\infty} e^{-\rho(y-x)} p(y) d y .
$$

We can rewrite equation (1) as

$$
\begin{aligned}
\phi_{\rho}(u) & =\sum_{n=1}^{\infty}\left(\phi_{\rho}(0)\right)^{n}\left(G_{\rho}^{(n-1) *}(u)-G_{\rho}^{n *}(u)\right) \\
& =\phi_{\rho}(0)+\sum_{n=1}^{\infty}\left(\left(\phi_{\rho}(0)\right)^{n+1} G_{\rho}^{n *}(u)-\left(\phi_{\rho}(0)\right)^{n} G_{\rho}^{n *}(u)\right)
\end{aligned}
$$

where $G_{\rho}^{0 *}(u)=1$ for $u \geq 0$ and equals 0 otherwise. Now define

$$
h_{\rho}(x)=\int_{x}^{\infty} e^{-\rho(y-x)} p(y) d y,
$$

and let

$$
H_{\rho}(x)=\int_{0}^{x} h_{\rho}(z) d z
$$

As $H_{\rho}(x)=m_{1} \tilde{p}_{1}(\rho) G_{\rho}(x)$, it follows that

$$
G_{\rho}^{n *}(u)=\frac{H_{\rho}^{n *}(u)}{\left(m_{1} \tilde{p}_{1}(\rho)\right)^{n}}
$$

where $H_{\rho}^{n^{*}}$ is the $n$-fold convolution of $H_{\rho}$ with itself, and hence

$$
\phi_{\rho}(u)=\frac{\lambda m_{1}}{c} \tilde{p}_{1}(\rho)+\sum_{n=1}^{\infty}\left(\frac{\lambda}{c}\right)^{n}\left(\frac{\lambda m_{1}}{c} \tilde{p}_{1}(\rho) H_{\rho}^{n^{*}}(u)-H_{\rho}^{n *}(u)\right) .
$$


Starting from equation (2.16) of Gerber and Shiu (1998), it is straightforward to show that

$$
\begin{aligned}
\tilde{\phi}_{\rho}(s) & =\int_{0}^{\infty} e^{-s u} \phi_{\rho}(u) d u \\
& =\frac{\psi(0)\left(\tilde{p}_{1}(s)-\tilde{p}_{1}(\rho)\right)}{\rho-s-\frac{\lambda}{c}(\tilde{p}(s)-\tilde{p}(\rho))} .
\end{aligned}
$$

Thus

$$
\tilde{\phi}_{\rho}(s)=\tilde{\phi}_{s}(\rho)=\int_{0}^{\infty} e^{-\rho u} \phi_{s}(u) d u
$$

and it is not difficult to see that $\phi_{\rho}$ is a Laplace transform with respect to $\rho$. In particular, we will demonstrate that

$$
\phi_{\rho}(u)=\int_{0}^{\infty} e^{-\rho t} \xi(u, t) d t
$$

and in Section 4 we explicitly identify $\xi(u, t)$. The function that we obtain on inversion is not, however, $w(u, t)$, as we must invert expression (3) with respect to $\delta$ to obtain that. However, as shown in the next section, there is a relationship between transforms with respect to $\rho$ and $\delta$, and we can exploit this relationship to obtain $w(u, t)$ from $\xi(u, t)$.

We conclude this section by remarking that in the special case when $P(x)=$ $1-e^{-\alpha x}$, we have $G_{\rho}(x)=P(x)$, and so

$$
G_{\rho}^{(n-1) *}(u)-G_{\rho}^{n *}(u)=e^{-\alpha u} \frac{(\alpha u)^{n-1}}{(n-1) !} .
$$

Thus, equation (2) becomes

$$
\phi_{\rho}(u)=\sum_{n=1}^{\infty} e^{-\alpha u} \frac{(\alpha u)^{n-1}}{(n-1) !}\left(\phi_{\rho}(0)\right)^{n},
$$

which is equation (2.2) of Drekic and Willmot (2003). Thus, for this individual claim amount distribution, the techniques that are presented in the next two sections are not required to obtain $w(u, t)$. We remark, however, that this special case is covered by the more general mixed Erlang example of Section 5.

\section{A TRANSFORM RELATIONSHIP}

As $\phi_{\rho}(0)=\psi(0) \tilde{p}_{1}(\rho)$, it follows that $\tilde{p}_{1}(\rho)$ is the Laplace transform (with respect to $\delta$ ) of the density function $w_{c}(0, t)$, so that

$$
\int_{0}^{\infty} e^{-p t} p_{1}(t) d t=\int_{0}^{\infty} e^{-\delta t} w_{c}(0, t) d t .
$$


In order to obtain an expression for $w_{c}(0, t)$ we require an expression for $e^{-p t}$ in terms of $\delta$, and, as noted in Lin and Willmot (1999), we can obtain this from Lagrange's implicit function theorem (see, for example, Goulden and Jackson (1983)). We restate this result as there is a typographical error in Lin and Willmot (1999): for any analytic function $\eta(z)$,

$\eta(\rho)=\eta\left(\frac{\delta+\lambda}{c}\right)+\left.\sum_{n=1}^{\infty}(-1)^{n} \frac{(\lambda / c)^{n}}{n !} \frac{d^{n-1}}{d z^{n-1}}\left\{\eta^{\prime}(z) \int_{0}^{\infty} e^{-z x} p^{n *}(x) d x\right\}\right|_{z=(\delta+\lambda) / c}$

where $p^{n *}$ is the $n$-fold convolution of the density $p$ with itself.

In particular, when $\eta(z)=e^{-z t}$, we have

$$
\begin{aligned}
e^{-\rho t}= & e^{-(\delta+\lambda) t / c} \\
& +\left.\sum_{n=1}^{\infty}(-1)^{n} \frac{(\lambda / c)^{n}}{n !}\left\{\frac{d^{n-1}}{d z^{n-1}}(-t) \int_{0}^{\infty} e^{-z(x+t)} p^{n *}(x) d x\right\}\right|_{z=(\delta+\lambda) / c} \\
= & e^{-(\delta+\lambda) t / c} \\
& +\left.\sum_{n=1}^{\infty}(-1)^{n} \frac{(\lambda / c)^{n}}{n !}\left\{(-1)^{n}(t) \int_{0}^{\infty}(x+t)^{n-1} e^{-z(x+t)} p^{n *}(x) d x\right\}\right|_{z=(\delta+\lambda) / c} \\
= & e^{-(\delta+\lambda) t / c} \\
& +\sum_{n=1}^{\infty} \frac{(\lambda / c)^{n}}{n !} t \int_{0}^{\infty}(x+t)^{n-1} e^{-(\delta+\lambda)(x+t) / c} p^{n *}(x) d x .
\end{aligned}
$$

Thus, for an arbitrary function $f$,

$$
\begin{aligned}
\tilde{f}(\rho)= & \int_{0}^{\infty} e^{-\rho t} f(t) d t \\
= & \int_{0}^{\infty} e^{-(\delta+\lambda) t / c} f(t) d t \\
& +\sum_{n=1}^{\infty} \frac{(\lambda / c)^{n}}{n !} \int_{0}^{\infty} t \int_{0}^{\infty}(x+t)^{n-1} e^{-(\delta+\lambda)(x+t) / c} p^{n *}(x) d x f(t) d t \\
= & c \int_{0}^{\infty} e^{-(\delta+\lambda) r} f(c r) d r \\
& +\sum_{n=1}^{\infty} \frac{(\lambda / c)^{n}}{n !} \int_{0}^{\infty} t \int_{t c}^{\infty} c^{n} r^{n-1} e^{-(\delta+\lambda) r} p^{n *}(c r-t) d r f(t) d t \\
= & c \int_{0}^{\infty} e^{-(\delta+\lambda) r} f(c r) d r \\
& +\sum_{n=1}^{\infty} \frac{\lambda^{n}}{n !} \int_{0}^{\infty} r^{n-1} e^{-(\delta+\lambda) r} \int_{0}^{c r} t p^{n *}(c r-t) f(t) d t d r \\
= & \int_{0}^{\infty} e^{-\delta t}\left(c e^{-\lambda t} f(c t)+\sum_{n=1}^{\infty} \frac{\lambda^{n}}{n !} t^{n-1} e^{-\lambda t} \int_{0}^{c t} y p^{n *}(c t-y) f(y) d y\right) d t
\end{aligned}
$$




$$
\begin{aligned}
& =\tilde{g}(\delta) \\
& =\int_{0}^{\infty} e^{-\delta t} g(t) d t
\end{aligned}
$$

where

$$
g(t)=c e^{-\lambda t} f(c t)+\sum_{n=1}^{\infty} \frac{\lambda^{n}}{n !} t^{n-1} e^{-\lambda t} \int_{0}^{c t} y p^{n *}(c t-y) f(y) d y .
$$

If we now return to the problem of determining $w_{c}(0, t)$, we see that the inverse with respect to $\delta$ of $\tilde{p}_{1}(\rho)$ is found by replacing $f$ by $p_{1}$ in equation (4). Hence

$$
w_{c}(0, t)=c e^{-\lambda t} p_{1}(c t)+\sum_{n=1}^{\infty} \frac{\lambda^{n}}{n !} t^{n-1} e^{-\lambda t} \int_{0}^{c t} y p^{n *}(c t-y) p_{1}(y) d y .
$$

This expression is implicit in the literature, but was derived in a different context. Dickson and dos Reis (1996, p. 152) give an expression for the density (which they denote by $a(t)$ ) of the time to recovery to surplus level 0 from the time of ruin given that ruin occurs from initial surplus $u \geq 0$. When $u=0$, a duality argument shows that the time to recovery is distributed as $T_{0, c}$. Setting $u=0$ in Dickson and dos Reis' formula for $a(t)$ yields formula (5) for $w_{c}(0, t)$ using the well-known fact (see, for example, Bowers et al (1997)) that $p_{1}$ is the density of the deficit at ruin when ruin occurs from initial surplus 0 .

\section{AnALysis WHen $u>0$}

Our aim in this section is to invert $\phi_{\rho}(u)$ with respect to $\rho$, then to use the transform relationship of the previous section to obtain $w(u, t)$. Thus, if

$$
\phi_{\rho}(u)=\int_{0}^{\infty} e^{-\rho t} \xi(u, t) d t,
$$

then it follows from equation (4) with $f(t)$ replaced by $\xi(u, t)$ and $g(t)$ by $w(u, t)$ that

$$
w(u, t)=c e^{-\lambda t} \xi(u, c t)+\sum_{n=1}^{\infty} \frac{\lambda^{n}}{n !} t^{n-1} e^{-\lambda t} \int_{0}^{c t} y p^{n *}(c t-y) \xi(u, y) d y .
$$

The remainder of this section is therefore devoted to finding $\xi(u, t)$ in equation (6), which may then be substituted into equation (7).

Our starting point is equation (3), namely

$$
\phi_{\rho}(u)=\frac{\lambda m_{1}}{c} \tilde{p}_{1}(\rho)+\sum_{n=1}^{\infty}\left(\frac{\lambda}{c}\right)^{n}\left(\frac{\lambda m_{1}}{c} \tilde{p}_{1}(\rho) H_{\rho}^{n *}(u)-H_{\rho}^{n *}(u)\right) .
$$

We can invert this with respect to $\rho$ on a term by term basis. Consider first inverting $H_{\rho}^{n^{*}}(u)$. For $n=1,2,3, \ldots$ let 


$$
H_{\rho}^{n^{*}}(u)=\int_{0}^{\infty} e^{-\rho t} b_{n}(u, t) d t
$$

To obtain an expression for $b_{n}(u, t)$ we first find an alternative expression for $H_{\rho}^{n^{*}}(u)$. From Dickson and Hipp (2001) we know that

$$
\int_{0}^{\infty} e^{-s u} h_{\rho}(u) d u=\frac{\tilde{p}(\rho)-\tilde{p}(s)}{s-\rho}
$$

so that for $n=1,2,3, \ldots$

$$
\int_{0}^{\infty} e^{-s u} h_{\rho}^{n *}(u) d u=\left(\frac{\tilde{p}(\rho)-\tilde{p}(s)}{s-\rho}\right)^{n}
$$

and hence

$$
\int_{0}^{\infty} e^{-s u} H_{\rho}^{n *}(u) d u=\frac{1}{S}\left(\frac{\tilde{p}(\rho)-\tilde{p}(s)}{s-\rho}\right)^{n}
$$

Now note that

$$
\begin{aligned}
\frac{1}{S}\left(\frac{\tilde{p}(\rho)-\tilde{p}(s)}{s-\rho}\right)^{n} & =\frac{(s-\rho)^{-n}}{s} \sum_{j=0}^{n}\left(\begin{array}{c}
n \\
j
\end{array}\right)(-1)^{j}[\tilde{p}(\rho)]^{n-j}[\tilde{p}(s)]^{j} \\
& =\frac{(s-\rho)^{-n}}{S}\left([\tilde{p}(\rho)]^{n}+\sum_{j=1}^{n}\left(\begin{array}{c}
n \\
j
\end{array}\right)(-1)^{j}[\tilde{p}(\rho)]^{n-j}[\tilde{p}(s)]^{j}\right),
\end{aligned}
$$

and that

$$
(s-\rho)^{-n}=\int_{0}^{\infty} e^{-s x} \frac{x^{n-1} e^{\rho x}}{\Gamma(n)} d x .
$$

Further, $s^{-1}[\tilde{p}(s)]^{j}$ is the Laplace transform (with respect to $s$ ) of $P^{j^{*}}(u)$ (which is the $j$-fold convolution of $P$ with itself, with $P^{0 *}(u)=1$ for $u \geq 0$ ). It therefore follows from equation (8) that

$$
\begin{aligned}
H_{\rho}^{n *}(u)= & {[\tilde{p}(\rho)]^{n} \int_{0}^{u} \frac{x^{n-1} e^{\rho x}}{\Gamma(n)} d x } \\
& +\sum_{j=1}^{n}\left(\begin{array}{c}
n \\
j
\end{array}\right)(-1)^{j} \frac{[\tilde{p}(\rho)]^{n-j}}{\Gamma(n)} \int_{0}^{u} x^{n-1} e^{\rho x} P^{j *}(u-x) d x \\
= & \frac{(-1)^{n}}{\Gamma(n)} \int_{0}^{u} x^{n-1} e^{\rho x} P^{n *}(u-x) d x \\
& +\sum_{j=0}^{n-1}\left(\begin{array}{c}
n \\
j
\end{array}\right)(-1)^{j} \frac{[\tilde{p}(\rho)]^{n-j}}{\Gamma(n)} \int_{0}^{u} x^{n-1} e^{\rho x} P^{j *}(u-x) d x .
\end{aligned}
$$


To invert this with respect to $\rho$, note that

$$
\begin{aligned}
& e^{-\rho u} H_{\rho}^{n *}(u) \\
& =\frac{(-1)^{n}}{\Gamma(n)} \int_{0}^{u} x^{n-1} e^{-\rho(u-x)} P^{n^{*}}(u-x) d x \\
& +\sum_{j=0}^{n-1}\left(\begin{array}{c}
n \\
j
\end{array}\right)(-1)^{j} \frac{[\tilde{p}(\rho)]^{n-j}}{\Gamma(n)} \int_{0}^{u} x^{n-1} e^{-\rho(u-x)} P^{j *}(u-x) d x \\
& =\frac{(-1)^{n}}{\Gamma(n)} \int_{0}^{u}(u-t)^{n-1} e^{-\rho t} P^{n^{*}}(t) d t \\
& +\sum_{j=0}^{n-1}\left(\begin{array}{l}
n \\
j
\end{array}\right)(-1)^{j} \frac{[\tilde{p}(\rho)]^{n-j}}{\Gamma(n)} \int_{0}^{u}(u-x)^{n-1} e^{-\rho x} P^{j *}(x) d x \\
& =\frac{(-1)^{n}}{\Gamma(n)} \int_{0}^{u}(u-t)^{n-1} e^{-\rho t} P^{n *}(t) d t \\
& +\sum_{j=0}^{n-1}\left(\begin{array}{c}
n \\
j
\end{array}\right) \frac{(-1)^{j}}{\Gamma(n)} \int_{0}^{u}(u-x)^{n-1} P^{j *}(x) \int_{0}^{\infty} e^{-\rho(t+x)} p^{(n-j) *}(t) d t d x \\
& =\frac{(-1)^{n}}{\Gamma(n)} \int_{0}^{u}(u-t)^{n-1} e^{-\rho t} P^{n *}(t) d t \\
& +\sum_{j=0}^{n-1}\left(\begin{array}{c}
n \\
j
\end{array}\right) \frac{(-1)^{j}}{\Gamma(n)} \int_{0}^{u}(u-x)^{n-1} P^{j *}(x) \int_{x}^{\infty} e^{-\rho v} p^{(n-j) *}(v-x) d v d x \\
& =\frac{(-1)^{n}}{\Gamma(n)} \int_{0}^{u} e^{-\rho t}(u-t)^{n-1} P^{n^{*}}(t) d t \\
& +\sum_{j=0}^{n-1}\left(\begin{array}{c}
n \\
j
\end{array}\right) \frac{(-1)^{j}}{\Gamma(n)} \int_{0}^{u} e^{-\rho t} \int_{0}^{t}(u-x)^{n-1} P^{j *}(x) p^{(n-j) *}(t-x) d x d t \\
& +\sum_{j=0}^{n-1}\left(\begin{array}{l}
n \\
j
\end{array}\right) \frac{(-1)^{j}}{\Gamma(n)} \int_{u}^{\infty} e^{-\rho t} \int_{0}^{u}(u-x)^{n-1} P^{j^{*}}(x) p^{(n-j) *}(t-x) d x d t \\
& =\frac{(-1)^{n}}{\Gamma(n)} \int_{0}^{u} e^{-\rho t}(u-t)^{n-1} P^{n *}(t) d t \\
& +\sum_{j=0}^{n-1}\left(\begin{array}{c}
n \\
j
\end{array}\right) \frac{(-1)^{j}}{\Gamma(n)} \int_{0}^{u} e^{-\rho t} \int_{0}^{t}(u-x)^{n-1} P^{j *}(x) p^{(n-j) *}(t-x) d x d t \\
& +\sum_{j=0}^{n-1}\left(\begin{array}{c}
n \\
j
\end{array}\right) \frac{(-1)^{j}}{\Gamma(n)} \int_{0}^{\infty} e^{-\rho(t+u)} \int_{0}^{u}(u-x)^{n-1} P^{j *}(x) p^{(n-j) *}(t+u-x) d x d t .
\end{aligned}
$$

As

$$
e^{-\rho u} H_{\rho}^{n *}(u)=\int_{0}^{\infty} e^{-\rho(t+u)} b_{n}(u, t) d t
$$


it follows by the uniqueness of the Laplace transformation that the coefficients of $e^{-\rho(t+u)}$ on both sides of this equation must be equal for all $t>-u$. In particular, for $t>0$, it follows that

$$
b_{n}(u, t)=\sum_{j=0}^{n-1}\left(\begin{array}{c}
n \\
j
\end{array}\right) \frac{(-1)^{j}}{\Gamma(n)} \int_{0}^{u}(u-x)^{n-1} P^{j^{*}}(x) p^{(n-j) *}(t+u-x) d x .
$$

Finally, inversion of equation (3) with respect to $\rho$ gives

$$
\xi(u, t)=\frac{\lambda}{c} \bar{P}(t)+\sum_{n=1}^{\infty}\left(\frac{\lambda}{c}\right)^{n}\left(\frac{\lambda}{c} \int_{0}^{t} \bar{P}(x) b_{n}(u, t-x) d x-b_{n}(u, t)\right) .
$$

\section{Mixed ERlang Claim amounts}

In this section we apply the results of the previous section when the individual claim amount distribution is a mixture of Erlang distributions with the same scale parameter. Thus,

$$
p(x)=\sum_{i=1}^{\infty} q_{i} \frac{\beta^{i} x^{i-1}}{(i-1) !} e^{-\beta x}=e^{-\beta x} \sum_{i=1}^{\infty} q_{i} \frac{\beta^{i}}{(i-1) !} x^{i-1},
$$

and hence

$$
P(x)=1-e^{-\beta x} \sum_{k=0}^{\infty} \bar{Q}_{k} \frac{(\beta x)^{k}}{k !}=e^{-\beta x} \sum_{k=0}^{\infty} Q_{k} \frac{\beta^{k}}{k !} x^{k}
$$

where

$$
Q_{k}=1-\bar{Q}_{k}=\sum_{i=1}^{k} q_{i}
$$

and we note that $Q_{0}=0$. Then

$$
\tilde{p}(s)=\sum_{i=1}^{\infty} q_{i}\left(\frac{\beta}{\beta+s}\right)^{i}
$$

and hence

$$
[\tilde{p}(s)]^{j}=\sum_{i=1}^{\infty} q_{i}^{j *}\left(\frac{\beta}{\beta+s}\right)^{i}
$$

where the coefficients $\left\{q_{i}^{j *}\right\}_{i=1}^{\infty}$ are obtained from the relationship

$$
\left(\sum_{i=1}^{\infty} q_{i} z^{i}\right)^{j}=\sum_{i=1}^{\infty} q_{i}^{j *} z^{i}
$$


and we adopt the notational convention that $q_{i}^{j *}=0$ for $i<j$. It then follows that

$$
p^{j *}(x)=e^{-\beta x} \sum_{i=1}^{\infty} q_{i}^{j *} \frac{\beta^{i}}{(i-1) !} x^{i-1}
$$

and

$$
P^{j *}(x)=e^{-\beta x} \sum_{k=0}^{\infty} Q_{k}^{j *} \frac{\beta^{k}}{k !} x^{k}
$$

where $Q_{k}^{0^{*}}=1$ and for $j=1,2,3, \ldots, Q_{k}^{j *}=\sum_{i=1}^{k} q_{i}^{j *}$.

We now write $b_{n}(u, t)$ as

$$
b_{n}(u, t)=\sum_{j=0}^{n-1}\left(\begin{array}{l}
n \\
j
\end{array}\right) \frac{(-1)^{j}}{\Gamma(n)} \sigma_{n, j}(u, t)=n \sum_{j=0}^{n-1} \frac{(-1)^{j}}{j !(n-j) !} \sigma_{n, j}(u, t)
$$

where

$$
\begin{aligned}
\sigma_{n, j}(u, t) & =\int_{0}^{u}(u-x)^{n-1} P^{j *}(x) p^{(n-j) *}(t+u-x) d x \\
& =\int_{0}^{u} x^{n-1} P^{j *}(u-x) p^{(n-j) *}(t+x) d x .
\end{aligned}
$$

Noting that

$$
\begin{aligned}
p^{(n-j)^{*}(t+x)} & =e^{-\beta(t+x)} \sum_{i=1}^{\infty} q_{i}^{(n-j)^{*}} \frac{\beta^{i}}{(i-1) !}(t+x)^{i-1} \\
& =e^{-\beta(t+x)} \sum_{i=1}^{\infty} q_{i}^{(n-j)^{*}} \frac{\beta^{i}}{(i-1) !} \sum_{m=0}^{i-1}\left(\begin{array}{c}
i-1 \\
m
\end{array}\right) t^{m} x^{i-1-m} \\
& =e^{-\beta(t+x)} \sum_{i=1}^{\infty} q_{i}^{(n-j)^{*}} \beta^{i} \sum_{m=0}^{i-1} \frac{t^{m}}{(i-1-m) ! m !} x^{i-1-m}
\end{aligned}
$$

we obtain

$$
\begin{aligned}
& \sigma_{n, j}(u, t) \\
& =\int_{0}^{u} x^{n-1} e^{-\beta(u-x)}\left\{\sum_{k=0}^{\infty} Q_{k}^{j *} \frac{\beta^{k}}{k !}(u-x)^{k}\right\} e^{-\beta(t+x)} \\
& \quad \times\left\{\sum_{i=1}^{\infty} q_{i}^{(n-j)^{*}} \beta^{i} \sum_{m=0}^{i-1} \frac{t^{m}}{(i-1-m) ! m !} x^{i-1-m}\right\} d x \\
& =e^{-\beta(u+t)} \sum_{i=1}^{\infty} q_{i}^{(n-j)^{*}} \beta^{i} \sum_{m=0}^{i-1} \frac{t^{m}}{(i-1-m) ! m !}
\end{aligned}
$$




$$
\begin{aligned}
& \times \sum_{k=0}^{\infty} Q_{k}^{j *} \frac{\beta^{k}}{k !} \int_{0}^{u} x^{n+i-m-2}(u-x)^{k} d x \\
= & e^{-\beta(u+t)} \sum_{i=1}^{\infty} q_{i}^{(n-j)^{*}} \beta^{i} \sum_{m=0}^{i-1} \frac{t^{m}}{(i-1-m) ! m !} \\
& \times \sum_{k=0}^{\infty} Q_{k}^{j *} \frac{\beta^{k}}{k !} u^{n+i-1-m+k} \int_{0}^{1} v^{n+i-m-2}(1-v)^{k} d v \\
= & e^{-\beta(u+t)} \sum_{i=1}^{\infty} q_{i}^{(n-j)^{*}} \beta^{i} \sum_{m=0}^{i-1} \frac{t^{m}}{(i-1-m) ! m !} \\
& \times \sum_{k=0}^{\infty} Q_{k}^{j *} \frac{\beta^{k}}{k !} u^{n+i-1-m+k} \frac{(n+i-m-2) ! k !}{(n+i-1-m+k) !} \\
= & e^{-\beta(u+t)} \sum_{i=1}^{\infty} q_{i}^{(n-j)^{*}} \beta^{i} \sum_{m=0}^{i-1} \frac{(n+i-m-2) !}{(i-1-m) ! m !} \\
& \times\left\{\sum_{k=0}^{\infty} Q_{k}^{j *} \beta^{k} \frac{u^{n+i-1-m+k}}{(n+i-1-m+k) !}\right\} t^{m} \\
= & e^{-\beta(u+t)} \sum_{m=0}^{\infty} \frac{t^{m}}{m !} \sum_{i=m+1}^{\infty} q_{i}^{(n-j)^{*}} \beta^{i} \frac{(n+i-m-2) !}{(i-1-m) !} \\
& \times \sum_{k=0}^{\infty} Q_{k}^{j *} \beta^{k} \frac{u^{n+i-1-m+k}}{(n+i-1-m+k) !} \\
= & e^{-\beta(u+t)} \sum_{m=0}^{\infty} \frac{t^{m}}{m !} \sum_{i=0}^{\infty} q_{i+m+1}^{(n-j) *} \beta^{i+m+1} \frac{(n+i-1) !}{i !} \sum_{k=0}^{\infty} Q_{k}^{j *} \beta^{k} \frac{u^{n+i+k}}{(n+i+k) !} .
\end{aligned}
$$

Thus, we can write

$$
\sigma_{n, j}(u, t)=e^{-\beta t} \sum_{m=0}^{\infty} \alpha_{n, j, m}(u) \frac{t^{m}}{m !}
$$

where

$$
\alpha_{n, j, m}(u)=e^{-\beta u} \sum_{i=0}^{\infty} q_{i+m+1}^{(n-j)^{*}} \frac{(n+i-1) !}{i !} \sum_{k=0}^{\infty} Q_{k}^{j *} \beta^{i+m+k+1} \frac{u^{n+i+k}}{(n+i+k) !} .
$$

Hence,

$$
\begin{aligned}
b_{n}(u, t) & =n e^{-\beta t} \sum_{j=0}^{n-1} \frac{(-1)^{j}}{j !(n-j) !} \sum_{m=0}^{\infty} \alpha_{n, j, m}(u) \frac{t^{m}}{m !} \\
& =e^{-\beta t} \sum_{m=0}^{\infty}\left\{n \sum_{j=0}^{n-1} \frac{(-1)^{j}}{j !(n-j) !} \alpha_{n, j, m}(u)\right\} \frac{t^{m}}{m !}
\end{aligned}
$$


so that we can write

$$
b_{n}(u, t)=e^{-\beta t} \sum_{m=0}^{\infty} \gamma_{n, m}(u) \frac{t^{m}}{m !}
$$

where

$$
\gamma_{n, m}(u)=n \sum_{j=0}^{n-1} \frac{(-1)^{j}}{j !(n-j) !} \alpha_{n, j, m}(u) .
$$

Next, we have

$$
\begin{aligned}
& \int_{0}^{t} \bar{P}(x) b_{n}(u, t-x) d x \\
= & \int_{0}^{t} e^{-\beta x}\left\{\sum_{k=0}^{\infty} \bar{Q}_{k} \frac{(\beta x)^{k}}{k !}\right\} e^{-\beta(t-x)}\left\{\sum_{m=0}^{\infty} \gamma_{n, m}(u) \frac{(t-x)^{m}}{m !}\right\} d x \\
= & e^{-\beta t} \sum_{k=0}^{\infty} \bar{Q}_{k} \frac{\beta^{k}}{k !} \sum_{m=0}^{\infty} \frac{\gamma_{n, m}(u)}{m !} \int_{0}^{t} x^{k}(t-x)^{m} d x \\
= & e^{-\beta t} \sum_{k=0}^{\infty} \bar{Q}_{k} \frac{\beta^{k}}{k !} \sum_{m=0}^{\infty} \frac{\gamma_{n, m}(u)}{m !} t^{k+m+1} \frac{k ! m !}{(k+m+1) !} \\
= & e^{-\beta t} \sum_{k=0}^{\infty} \bar{Q}_{k} \beta^{k} \sum_{m=0}^{\infty} \gamma_{n, m}(u) \frac{t^{k+m+1}}{(k+m+1) !} \\
= & e^{-\beta t} \sum_{k=0}^{\infty} \bar{Q}_{k} \beta^{k} \sum_{m=k+1}^{\infty} \gamma_{n, m-k-1}(u) \frac{t^{m}}{m !} \\
= & e^{-\beta t} \sum_{m=1}^{\infty} \frac{t^{m}}{m !} \sum_{k=0}^{m-1} \bar{Q}_{k} \beta^{k} \gamma_{n, m-k-1}(u) .
\end{aligned}
$$

Therefore,

$$
\begin{aligned}
& \frac{\lambda}{c} \int_{0}^{t} \bar{P}(x) b_{n}(u, t-x) d x-b_{n}(u, t) \\
= & \frac{\lambda}{c} e^{-\beta t} \sum_{m=1}^{\infty} \frac{t^{m}}{m !} \sum_{k=0}^{m-1} \bar{Q}_{k} \beta^{k} \gamma_{n, m-k-1}(u)-e^{-\beta t} \sum_{m=0}^{\infty} \gamma_{n, m}(u) \frac{t^{m}}{m !} \\
= & e^{-\beta t} \sum_{m=1}^{\infty} \frac{t^{m}}{m !}\left(\frac{\lambda}{c} \sum_{k=0}^{m-1} \bar{Q}_{k} \beta^{k} \gamma_{n, m-k-1}(u)-\gamma_{n, m}(u)\right)-e^{-\beta t} \gamma_{n, 0}(u) \\
= & e^{-\beta t} \sum_{m=0}^{\infty} \frac{t^{m}}{m !} a_{n, m}(u)
\end{aligned}
$$

where $a_{n, 0}(u)=-\gamma_{n, 0}(u)$ and for $m=1,2,3, \ldots$ 


$$
a_{n, m}(u)=\frac{\lambda}{c} \sum_{k=0}^{m-1} \bar{Q}_{k} \beta^{k} \gamma_{n, m-k-1}(u)-\gamma_{n, m}(u) .
$$

Thus,

$$
\begin{aligned}
\xi(u, t) & =\frac{\lambda}{c} \bar{P}(t)+\sum_{n=1}^{\infty}\left(\frac{\lambda}{c}\right)^{n}\left(\frac{\lambda}{c} \int_{0}^{t} \bar{P}(x) b_{n}(u, t-x) d x-b_{n}(u, t)\right) \\
& =\frac{\lambda}{c} e^{-\beta t} \sum_{m=0}^{\infty} \bar{Q}_{m} \frac{(\beta t)^{m}}{m !}+\sum_{n=1}^{\infty}\left(\frac{\lambda}{c}\right)^{n}\left(e^{-\beta t} \sum_{m=0}^{\infty} \frac{t^{m}}{m !} a_{n, m}(u)\right) .
\end{aligned}
$$

We can write this in a condensed form as

$$
\xi(u, t)=e^{-\beta t} \sum_{m=0}^{\infty} \tau_{m}(u) \frac{t^{m}}{m !}
$$

where

$$
\tau_{0}(u)=\frac{\lambda}{c}+\sum_{n=1}^{\infty}\left(\frac{\lambda}{c}\right)^{n} a_{n, 0}(u)
$$

and for $m=1,2,3, \ldots$,

$$
\tau_{m}(u)=\frac{\lambda}{c} \bar{Q}_{m} \beta^{m}+\sum_{n=1}^{\infty}\left(\frac{\lambda}{c}\right)^{n} a_{n, m}(u) .
$$

To obtain $w(u, t)$, we next require

$$
\begin{aligned}
& \int_{0}^{c t} y p^{n *}(c t-y) \xi(u, y) d y \\
= & \int_{0}^{c t} e^{-\beta(c t-y)}\left\{\sum_{i=1}^{\infty} q_{i}^{n *} \frac{\beta^{i}}{(i-1) !}(c t-y)^{i-1}\right\} e^{-\beta y}\left\{\sum_{m=0}^{\infty} \tau_{m}(u) \frac{y^{m+1}}{m !}\right\} d y \\
= & e^{-c \beta t} \sum_{i=1}^{\infty} q_{i}^{n *} \frac{\beta^{i}}{(i-1) !} \sum_{m=0}^{\infty} \frac{\tau_{m}(u)}{m !} \int_{0}^{c t}(c t-y)^{i-1} y^{m+1} d y \\
= & e^{-c \beta t} \sum_{i=1}^{\infty} q_{i}^{n *} \frac{\beta^{i}}{(i-1) !} \sum_{m=0}^{\infty} \frac{\tau_{m}(u)}{m !}(c t)^{i+m+1} \frac{(i-1) !(m+1) !}{(i+m+1) !} \\
= & e^{-c \beta t} \sum_{i=1}^{\infty} q_{i}^{n *} \beta^{i} \sum_{m=0}^{\infty} \tau_{m}(u) \frac{(c t)^{i+m+1}}{(i+m+1) !}(m+1) \\
= & e^{-c \beta t} \sum_{i=1}^{\infty} q_{i}^{n *} \beta^{i} \sum_{m=i+1}^{\infty}(m-i) \tau_{m-i-1}(u) \frac{(c t)^{m}}{m !} \\
= & e^{-c \beta t} \sum_{m=2}^{\infty} \frac{(c t)^{m}}{m !} \sum_{i=1}^{m-1} q_{i}^{n *} \beta^{i}(m-i) \tau_{m-i-1}(u) .
\end{aligned}
$$


Inserting this in equation (7) for $w(u, t)$, we obtain

$$
\begin{aligned}
& w(u, t) \\
& =c e^{-\lambda t} \xi(u, c t)+\sum_{n=1}^{\infty} \frac{\lambda^{n}}{n !} t^{n-1} e^{-\lambda t} \int_{0}^{c t} y p^{n *}(c t-y) \xi(u, y t) d y \\
& =e^{-(\lambda+c \beta) t}\left(c \sum_{m=0}^{\infty} \tau_{m}(u) \frac{(c t)^{m}}{m !}\right. \\
& \left.+\sum_{n=1}^{\infty} \frac{\lambda^{n}}{n !} t^{n-1} \sum_{m=2}^{\infty} \frac{(c t)^{m}}{m !} \sum_{i=1}^{m-1} q_{i}^{n *} \beta^{i}(m-i) \tau_{m-i-1}(u)\right) \\
& =c e^{-(\lambda+c \beta) t}\left(\sum_{m=0}^{\infty} \tau_{m}(u) \frac{(c t)^{m}}{m !}\right. \\
& \left.+\sum_{n=1}^{\infty} \frac{(\lambda / c)^{n}}{n !}(c t)^{n-1} \sum_{m=2}^{\infty} \frac{(c t)^{m}}{m !} \sum_{i=1}^{m-1} q_{i}^{n *} \beta^{i}(m-i) \tau_{m-i-1}(u)\right) \\
& =c e^{-(\lambda+c \beta) t}\left(\sum_{m=0}^{\infty} \tau_{m}(u) \frac{(c t)^{m}}{m !}\right. \\
& \left.+\sum_{m=2}^{\infty} \frac{(c t)^{m}}{m !} \sum_{n=1}^{\infty} \frac{(\lambda / c)^{n}}{n !}(c t)^{n-1} \sum_{i=1}^{m-1} q_{i}^{n *} \beta^{i}(m-i) \tau_{m-i-1}(u)\right) .
\end{aligned}
$$

We remark that the above derivation is tedious rather than complicated. However, an important point about this derivation is that the result is expressed in terms of simple functions of $u$ and $t$ and is therefore easy to program to obtain numerical values of the density given a set of parameter values.

Further, it is a straightforward exercise to integrate this density to obtain a formula for $\psi(u, t)$. Specifically,

$$
\begin{aligned}
\psi(u, t)= & \sum_{m=0}^{\infty} \frac{\tau_{m}(u) c^{m+1}}{(\lambda+c \beta)^{m+1}} E(m+1,(\lambda+c \beta) t) \\
& +\sum_{m=2}^{\infty} \frac{c^{m}}{m !} \sum_{n=1}^{\infty} \frac{\lambda^{n}}{n !} \frac{(m+n-1) !}{(\lambda+c \beta)^{m+n}} E(m+n,(\lambda+c \beta) t) \\
& \times \sum_{i=1}^{m-1} q_{i}^{n *} \beta^{i}(m-i) \tau_{m-i-1}(u)
\end{aligned}
$$

where for $n=1,2,3, \ldots$,

$$
E(n, x)=1-e^{-x} \sum_{j=0}^{n-1} \frac{x^{j}}{j !}
$$

Tables 1 and 2 show values of $\psi(1, t)$ and $\psi(10, t)$ respectively for some values of $t$ when $\theta=0.1$ and the individual claim amount distribution is $\operatorname{Erlang}(n, n)$ 
TABLE 1

VALUES OF $\psi(1, t)$

\begin{tabular}{rcccc}
\hline \hline $\boldsymbol{t}$ & $\boldsymbol{n = 1}$ & $\boldsymbol{n = 2}$ & $\boldsymbol{n = 3}$ & $\boldsymbol{n = 4}$ \\
\hline 2 & 0.3546 & 0.3649 & 0.3669 & 0.3671 \\
4 & 0.4753 & 0.4830 & 0.4840 & 0.4839 \\
6 & 0.5404 & 0.5459 & 0.5462 & 0.5457 \\
8 & 0.5826 & 0.5863 & 0.5860 & 0.5852 \\
10 & 0.6126 & 0.6150 & 0.6142 & 0.6132 \\
20 & 0.6906 & 0.6889 & 0.6866 & 0.6849 \\
40 & 0.7471 & 0.7416 & 0.7379 & 0.7354 \\
\hline \hline
\end{tabular}

TABLE 2

VALUES OF $\psi(10, t)$

\begin{tabular}{rcccc}
\hline \hline $\boldsymbol{t}$ & $\boldsymbol{n}=\mathbf{1}$ & $\boldsymbol{n}=\mathbf{2}$ & $\boldsymbol{n}=\mathbf{3}$ & $\boldsymbol{n}=\mathbf{4}$ \\
\hline 2 & 0.0013 & 0.0002 & 0.0000 & 0.0000 \\
4 & 0.0059 & 0.0014 & 0.0006 & 0.0004 \\
6 & 0.0131 & 0.0042 & 0.0023 & 0.0015 \\
8 & 0.0220 & 0.0084 & 0.0050 & 0.0037 \\
10 & 0.0319 & 0.0137 & 0.0088 & 0.0067 \\
20 & 0.0822 & 0.0464 & 0.0345 & 0.0289 \\
40 & 0.1573 & 0.1038 & 0.0840 & 0.0740 \\
\hline \hline
\end{tabular}

for $n=1,2,3,4$. The Erlang $(n, n)$ distribution is just a special case of our mixed Erlang distribution, obtained by setting $q_{n}=1$ and $q_{i}=0$ for $i \neq n$, and $\beta=n$ (which makes the distribution's mean 1). Some of the values in these tables already exist in the literature, e.g. Seal (1978) for the case $n=1$ and Garcia (2002) for the case $n=2$, but the values for $n=3$ and $n=4$ are all new. We remark that in the case $n=3$, our calculations show that some of the approximations to $\psi(u, t)$ in a recent paper by Asmussen et al (2002) are very accurate.

\section{ACKNOWLEDGEMENTS}

Support for Gordon Willmot from the Munich Reinsurance Company is gratefully acknowledged, as is support from the Natural Sciences and Engineering Research Council of Canada.

\section{REFERENCES}

Asmussen, S. (2000) Ruin probabilities. World Scientific, Singapore.

Asmussen, S., Avram, F. and Usabel, M. (2002) Erlangian approximations for finite-horizon ruin probabilities. ASTIN Bulletin 32, 267-281. 
Bowers, N.L., Gerber, H.U., Hickman, J.C., Jones, D.A. and Nesbitt, C.J. (1997) Actuarial Mathematics, 2nd edition. Society of Actuaries, Itasca, IL.

Dickson, D.C.M. and Dos Reis, A.D.E. (1996) On the distribution of the duration of negative surplus. Scandinavian Actuarial Journal, 148-164.

Dickson, D.C.M. and Hipp, C. (2001) On the time to ruin for Erlang(2) risk processes. Insurance: Mathematics \& Economics 29, 333-344.

Dickson, D.C.M., Hughes, B. and Zhang, L. (2003) The density of the time to ruin for a Sparre Andersen process with Erlang arrivals and exponential claims. Centre for Actuarial Studies Research Paper Series No. 111, University of Melbourne.

Dickson, D.C.M. and WATERS, H.R. (2002) The distribution of the time to ruin in the classical risk model. ASTIN Bulletin 32, 299-313.

Drekic, S., Stafford J.E. and Willmot, G.E. (2004) Symbolic calculation of the moments of the time to ruin. Insurance: Mathematics \& Economics 34, 109-120.

Drekic, S. and Willmot, G.E. (2003) On the density and moments of the time to ruin with exponential claims. ASTIN Bulletin 33, 11-21.

GARCIA, J.M. (2002) Explicit solutions for survival probabilities in a finite time horizon. Presented to the 6th International IME Conference, Lisbon. http://pascal.iseg.utl.pt/ ${ }^{\sim}$ cemapre/ ime2002/index.html (No. 49).

Gerber, H.U. and ShiU, E.S.W. (1998) On the time value of ruin. North American Actuarial Journal 2(1), 48-78.

Goulden, I.P and JACKSON, D.M. (1983) Combinatorial Enumeration. Wiley, New York.

Lin, X.S. and Willmot, G.E. (1999) Analysis of a defective renewal equation arising in ruin theory. Insurance: Mathematics \& Economics 25, 63-84.

Lin, X.S. and Willmot, G.E. (2000) The moments of the time to ruin, the surplus before ruin and the deficit at ruin. Insurance: Mathematics \& Economics 27, 19-44.

SEAL, H.L. (1978) Survival Probabilities. John Wiley and Sons, New York.

\author{
DAVID C.M. Dickson \\ Centre for Actuarial Studies \\ Department of Economics \\ University of Melbourne \\ Victoria 3010 \\ Australia
}

Gordon E. WiLLMoT

Department of Statistics and Actuarial Science

University of Waterloo

Waterloo

Ontario

Canada N2L 3GI 